\title{
Flow Induced by Torsional Oscillations of a Disk in a Rotating Visco-Elastic Fluid
}

\author{
S. Das \\ Department of \\ Mathematics, University \\ of Gour Banga, Malda \\ 731 103, India
}

\author{
S. L. Maji \\ Department of \\ Applied Mathematics, \\ Vidyasagar University \\ Midnapore 721 102, \\ India
}

\author{
R. N. Jana \\ Department of \\ Applied Mathematics, \\ Vidyasagar University, \\ Midnapore 721 102, \\ India
}

\author{
G. S. Seth \\ Department of \\ Mathematics, \\ Indian School of Mines \\ University, Dhanbad, \\ India
}

\begin{abstract}
The flow due to torsional oscillations of a disk about a steady rotation in an elastico-viscous fluid which is also rotating has been analyzed. The flow is characterized by two circularly polarized waves travelling with different velocities. It is found that the depth of penetration or wave length decreases for $0 \leq k \sigma \leq 0.58$ and increases for $k \sigma>0.59$ where $k$ is the elastic parameter and $\sigma$ is the frequency parameter. The unsteady axial velocity at large distance from the disk has a phase lead for $\sigma<2$ and a phase lag for $\sigma>2$.

Key words: Visco-elastic, torsional oscillations, torque.
\end{abstract}

\section{INTRODUCTION}

The torsional oscillations of an infinite disk in an incompressible viscous fluid has been studied by Rosenblat[1]. On the other hand, the flow caused by torsional oscillations for a disc with frequency $n^{*}$ about a state of steady rotation in a viscous fluid which is also rotating with angular velocity was investigated by Henney [2]. The flow induced by a fluid between two infinite disks when one disk is at rest and the other disk performs small torsional oscillations about their common axis for second order fluids have been studied by Bhatnagar and Rajeswari [3] and Srivastava [4]. The elastico-viscous liquid $B^{\prime}$ characterized by Walters [5] has been found to have a close resemblance so real fluids, like oils, blood and high polymer solutions. Basu and Debnath [6] have studied the unsteady flow of elastico-viscous fluid induced by torsional oscillations of a plate. Rajagopal [7] have discussed the flow of visco-elastic fluids between rotating disks.

In the present paper, we have studied the flow of a visco-elastic fluid $B^{\prime}$ induced by torsional oscillations of a disk in a rotating fluid. It is found that the flow is characterized by two-deck boundary layer such that one of the boundary layers tends to infinitely thickness when $\sigma \rightarrow 2, \sigma$ being frequency parameter. It is found that the axial velocity at a large distance from the disk has a phase lead for $\sigma<2$ and a phase lag for $\sigma>2$.

\section{MATHEMATICAL FORMULATION AND ITS SOLUTION}

The constitutive equations characterizing the elastico-viscous liquid $B^{\prime}$ are

$$
\begin{aligned}
& p_{i k}=-p g_{i k}+p_{i k}^{\prime}, \\
& p_{i k}^{\prime}=2 \int_{-\infty}^{t} \psi\left(t-t^{\prime}\right) \frac{\partial x^{i}}{\partial x^{\prime m}} \frac{\partial x^{k}}{\partial x^{\prime r}} e^{(1) m r} d t^{\prime},
\end{aligned}
$$

where $p_{i k}$ is the stress tensor, $p$ an arbitrary isotropic pressure, $g_{i k}$ the metric tensor, $e^{(l) m k}$ the rate of strain tensor and

$$
\psi\left(t-t^{\prime}\right)=\int_{0}^{\infty} \frac{N(\tau)}{\tau} \exp \left[-\frac{t}{\tau}\left(t-t^{\prime}\right)\right] d \tau
$$

where $N(\tau)$ is the distribution function of the relaxation time. Walters [5] has shown that in the case of liquids with short memories (i.e., short relaxation time) the above equations give

$$
p_{i k}^{\prime}=2 \eta_{0} e^{(1) i k}-2 k_{0} \frac{\delta e^{(1) i k}}{\delta t},
$$

where $\eta_{0}=\int_{0}^{\infty} N(\tau) d \tau$ is the limiting viscosity at small rate of shear, $k_{0}=\int_{0}^{\infty} \tau N(\tau) d \tau$ and $\delta / \delta t$ denotes the convected differentiation of a tensor.

In view of the above equations, the equation of continuity and the momentum are

$$
\begin{aligned}
\nabla \cdot \vec{q}=0, & \\
\frac{\partial \vec{q}}{\partial t}+(\vec{q} \cdot \nabla) \vec{q} & =-\nabla p+v \nabla^{2} \vec{q} \\
& -k_{0}^{*}\left[\frac{\partial}{\partial t} \nabla^{2} \vec{q}+2(\vec{q} \cdot \nabla) \nabla^{2} \vec{q}-\nabla^{2}\{(\vec{q} \cdot \nabla) \vec{q}\}\right],(6)
\end{aligned}
$$

where $\quad v=\frac{\eta_{0}}{\rho} \quad$ is the kinematic viscosity, $k_{0}^{*}=\frac{k_{0}}{\rho}$ the coefficient of elasticity and $\rho$ the fluid density.

A disk coinciding with the plane $z=0$ is rotating in unison with an elastico-viscous fluid with angular velocity $\Omega$ about an axis $z$. The disk also performs torsional oscillations with amplitude $\varepsilon$ and frequency $n^{*}$ so that the absolute angular velocity of the disk is $\Omega+\varepsilon n^{*} e^{i n^{*} t}$. The fluid occupying the region $z>0$ is also rotating with an angular velocity $\Omega$ at a large distance from the disk [see in Fig.1]. We introduce cylindrical polar coordinates $(r, \varphi, z)$ such that $\vec{q}$ has components $(u, v, w)$ along $(r, \varphi, z)$ directions respectively.

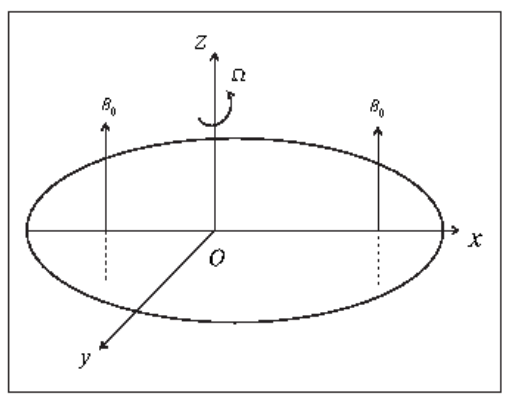

Figure 1. Geometry of the problem 
Substituting

$$
\begin{aligned}
& u=r f(z) e^{i n^{*} t}, v=r \Omega+r g(z) e^{i{ }^{*} t}, w=h(z) e^{i n^{*} t}, \\
& p=p_{0}+\frac{1}{2} \rho r^{2} \Omega^{2}+p_{1}(r, z) e^{i{ }^{*} t},
\end{aligned}
$$

in equations (5) and (6), we obtain following equations after linearization

$$
\begin{aligned}
& 2 f(z)+\frac{d h(z)}{d z}=0, \\
& i n^{*} f(z)-2 \Omega g(z)=-\frac{1}{\rho} \frac{\partial p_{1}}{\partial r}+\left(v-i n^{*} k_{0}^{*}\right) \frac{d^{2} f(z)}{d z^{2}},
\end{aligned}
$$

(9)

$$
\begin{aligned}
& i n^{*} g(z)+2 \Omega f(z)=\left(v-i n^{*} k_{0}^{*}\right) \frac{d^{2} g(z)}{d z^{2}}, \\
& i n^{*} h(z)=-\frac{1}{\rho} \frac{\partial p_{1}}{\partial z}+\left(v-i n^{*} k_{0}^{*}\right) \frac{d^{2} h(z)}{d z^{2}} .
\end{aligned}
$$

The linearization in equations (8) to (11) is valid provided the amplitude of the torsional oscillations is small such that $\varepsilon n^{*} \square \Omega$.

The boundary conditions are

$$
\begin{aligned}
& f=0, g=\varepsilon n^{*}, h=0 \text { at } z=0, \\
& f \rightarrow 0, g \rightarrow 0 \text { as } z \rightarrow \infty .
\end{aligned}
$$

Since $h(z)$ is a function of $z$ only, we have from (11) on differentiation with respect to $r$ as

$$
\frac{\partial^{2} p_{1}}{\partial r \partial z}=0 .
$$

Integrating the equation (13) with respect to $z$ and using (9) together with conditions $f \rightarrow 0$ and $g \rightarrow 0$ as $z \rightarrow \infty$, we have $\frac{\partial p_{1}}{\partial r}=0$.

Introducing

$$
f_{1,2}=f \pm i g,
$$

equations (9) and (10) become

$$
\frac{d^{2} F_{1,2}}{d z^{2}}-\frac{i\left(n^{*} \pm 2 \Omega\right)}{\left(v-i n^{*} k_{0}^{*}\right)} F_{1,2}=0 .
$$

The boundary conditions for $F_{1,2}$ are

$$
F_{1,2}= \pm i \varepsilon n^{*} \text { at } z=0 \text { and } F_{1,2} \rightarrow 0 \text { as } z \rightarrow \infty \text {. }
$$

The solution of (15) subject to the boundary conditions (16) is

$$
F_{1,2}= \pm i \varepsilon n^{*} \exp \left[-M_{1,2} z\right] \text {, }
$$

where

$$
M_{1,2}=\left\{i\left(n^{*} \pm 2 \Omega\right) /\left(v-i n^{*} k_{0}^{*}\right)\right\}^{\frac{1}{2}} .
$$

Introducing

$$
\eta=z\left(\frac{\Omega}{v}\right)^{\frac{1}{2}}, \sigma=\frac{n^{*}}{\Omega}, k=\frac{k_{0}^{*} \Omega}{v},
$$

and using (14), we get

$$
\begin{aligned}
& f=\frac{1}{2} i \varepsilon n^{*}\left[\exp \left\{-\left(\alpha_{1}+i \beta_{1}\right) \eta\right\}-\exp \left\{-\left(\alpha_{2,3} \pm i \beta_{2,3}\right) \eta\right\}\right], \\
& g=\frac{1}{2} \varepsilon n^{*}\left[\exp \left\{-\left(\alpha_{1}+i \beta_{1}\right) \eta\right\}+\exp \left\{-\left(\alpha_{2,3} \pm i \beta_{2,3}\right) \eta\right\}\right]
\end{aligned}
$$

Substituting $f$ given by (20) in the equation (8) and on integration after using (12), we get

$$
h=i \varepsilon n^{*}\left(\frac{v}{\Omega}\right)^{\frac{1}{2}}\left[\frac{e^{-\left(\alpha_{1}+i \beta_{1}\right) \eta}-1}{\alpha_{1}+i \beta_{1}}-\frac{e^{-\left(\alpha_{2,3} \pm i \beta_{2,3}\right) \eta}-1}{\alpha_{2,3} \pm i \beta_{2,3}}\right],
$$

where

$$
\begin{aligned}
& \alpha_{1,2}=\frac{1}{\sqrt{2}}\left(\frac{\sigma \pm 2}{1+k^{2} \sigma^{2}}\right)^{\frac{1}{2}}(a-b), \beta_{1,2}=\frac{1}{\sqrt{2}}\left(\frac{\sigma \pm 2}{1+k^{2} \sigma^{2}}\right)^{\frac{1}{2}}(a+b), \\
& \alpha_{3}, \beta_{3}=\frac{1}{\sqrt{2}}\left(\frac{2-\sigma}{1+k^{2} \sigma^{2}}\right)^{\frac{1}{2}}\left\{\left(1+k^{2} \sigma^{2}\right)^{\frac{1}{2}} \pm k \sigma\right\}^{\frac{1}{2}},
\end{aligned}
$$

$$
a, b=\frac{1}{\sqrt{2}}\left\{\left(1+k^{2} \sigma^{2}\right)^{\frac{1}{2}} \pm 1\right\}^{\frac{1}{2}} .
$$

In the above equations (20)-(22), the subscript ' 2 ' and plus sign are taken for $\sigma>2$ and the subscript ' 3 ' and negative sign are taken for $\sigma<2$.

Substituting (20)-(22) in (7), we have the following expressions for $u, v$ and $w$ in real forms

$u=\frac{1}{2} r \varepsilon n^{*}\left[e^{-\alpha_{2,3^{\eta}} \eta} \sin \left(\sigma \tau \mp \beta_{2,3} \eta\right)-e^{-\alpha_{1} \eta} \sin \left(\sigma \tau-\beta_{1} \eta\right)\right]$,

$v=r \Omega+\frac{1}{2} r \varepsilon n^{*}\left[e^{-\alpha_{1} \eta} \cos \left(\sigma \tau-\beta_{1} \eta\right)+e^{-\alpha_{2,3} \eta} \cos \left(\sigma \tau \mp \beta_{2,3} \eta\right)\right]$,

$w=\varepsilon n^{*}\left(\frac{v}{\Omega}\right)^{\frac{1}{2}} \times\left[\frac{e^{-\alpha_{1} \eta}}{\alpha_{1}^{2}+\beta_{1}^{2}}\left\{\beta_{1} \cos \left(\sigma \tau-\beta_{1} \eta\right)-\alpha_{1} \sin \left(\sigma \tau-\beta_{1} \eta\right)\right\}\right.$ $+\frac{e^{-\alpha_{2,3} \eta}}{\alpha_{2,3}^{2}+\beta_{2,3}^{2}}\left\{\alpha_{2,3} \sin \left(\sigma \tau \mp \beta_{2,3} \eta\right) \mp \beta_{2,3} \cos \left(\sigma \tau \mp \beta_{2,3} \eta\right)\right\}$

$$
+R \sin (\sigma \tau-\phi)] \text {, }
$$

where

$$
\begin{aligned}
& R=\left[\frac{2\left(1+k^{2} \sigma^{2}\right)^{1 / 2}}{\sigma^{2}-4}\left\{\sigma-\left(\sigma^{2}-4\right)^{\frac{1}{2}}\right\}^{1 / 2}\right]^{1 / 2}, \\
& \phi=\tan ^{-1}\left\{\left(1+k^{2} \sigma^{2}\right)^{1 / 2}+k \sigma\right\} \text { for } \sigma>2,
\end{aligned}
$$

and

$$
\begin{aligned}
& R=\left[\frac{\left(\alpha_{3}-\alpha_{1}\right)^{2}+\left(\beta_{3}-\beta_{1}\right)^{2}}{\left(\alpha_{1} \alpha_{3}+\beta_{1} \beta_{3}\right)^{2}+\left(\beta_{1} \alpha_{3}-\alpha_{1} \beta_{3}\right)^{2}}\right]^{1 / 2}, \\
& \tan \phi_{1}=\frac{\beta_{1}+\beta_{3}}{\alpha_{3}-\alpha_{1}} \tan \phi_{2}=\frac{\beta_{1} \alpha_{3}-\alpha_{1} \beta_{3}}{\alpha_{1} \alpha_{3}-\beta_{1} \beta_{3}} \\
& \text { and } \phi=\phi_{1}+\phi_{2} \text { for } \sigma<2 .
\end{aligned}
$$

Setting $u_{1}=\frac{2 u}{\varepsilon n^{*} r}, \quad v_{1}=\frac{2(v-\Omega r)}{\varepsilon n^{*} r}$ and $w_{1}=w / \varepsilon n^{*}\left(\frac{v}{\Omega}\right)^{\frac{1}{2}}$, equations (33)-(35) become

$$
u_{1}=e^{-\alpha_{2,3} \eta} \sin \left(\sigma \tau \mp \beta_{2,3} \eta\right)-e^{-\alpha_{1} \eta} \sin \left(\sigma \tau-\beta_{1} \eta\right),
$$




$$
\begin{aligned}
& v_{1}=e^{-\alpha_{1} \eta} \cos \left(\sigma \tau-\beta_{1} \eta\right)+e^{-\alpha_{2,3} \eta} \cos \left(\sigma \tau \mp \beta_{2,3} \eta\right), \\
& w_{1}=\frac{e^{-\alpha_{1} \eta}}{\alpha_{1}^{2}+\beta_{1}^{2}}\left\{\beta_{1} \cos \left(\sigma \tau-\beta_{1} \eta\right)-\alpha_{1} \sin \left(\sigma \tau-\beta_{1} \eta\right)\right\}, \\
& +\frac{e^{-\alpha_{2,3} \eta}}{\alpha_{2,3}^{2}+\beta_{2,3}^{2}}\left\{\alpha_{2,3} \sin \left(\sigma \tau \mp \beta_{2,3} \eta\right) \mp \beta_{2,3} \cos \left(\sigma \tau \mp \beta_{2,3} \eta\right)\right\} \\
& \quad+R \sin (\sigma \tau-\phi) .
\end{aligned}
$$

\section{RESULTS AND DISCUSSION}

Equations (24) and (25) show that for $\sigma>2$ the velocity consists of damped harmonic oscillations with amplitude $\frac{1}{2} r \varepsilon n^{*} e^{-\alpha_{1} \eta}$ and $\frac{1}{2} r \varepsilon n^{*} e^{-\alpha_{2} \eta}$ having phase lags $\beta_{1} \eta$ and $\beta_{2} \eta$ respectively relative to the disk. The depth of penetration or wave length of the two layers are $2 \pi(v / \Omega)^{\frac{1}{2}} \beta_{1}$ and $2 \pi(v / \Omega)^{\frac{1}{2}} \beta_{2}$ respectively. It is found from equation (23) that both $\beta_{1}$ and $\beta_{2}$ increase for $0 \leq k \sigma \leq 0.58$ and decrease for $k \sigma>0.58$ which implies that the depths of penetration decrease for $0 \leq k \sigma \leq 0.58$ and increase for $k \sigma>0.58$.

For $\sigma<2$ the velocity consists of damped harmonic oscillations of which one of the amplitude is the same as that for the case $\sigma>2$ and the second one has an amplitude $\frac{1}{2} r \varepsilon n^{*} e^{-\alpha_{3} \eta}$ and has a phase advance $\beta_{3} \eta$ with respect to the disk. The depth of penetration of this second layer is $2 \pi(v / \Omega)^{\frac{1}{2}} \beta_{3}$ which decreases with $k \sigma$.

For $\sigma=2$, a normal solution does not exist which is similar to the result obtained by Thornley [8] in her study of nontorsional oscillations of an infinite plate rotating in accordance with a viscous fluid. This is due to the fact that the whole liquid is affected by the motion of the plate and the oscillation is not confined to a well-defined Ekman layer near the disk.

To study the effects of frequency parameter on the velocity distributions we have presented the radial velocity $u_{1}$ and the azimuthal velocity $v_{1}$ against $\eta$ in Fig. 2 for several values of frequency parameter $\sigma$. It is observed from Fig.2 that the radial velocity $u_{1}$ decreases whereas the azimuthal velocity $v_{1}$ increases and they oscillates away from the disc with an increase in frequency parameter $\sigma$.

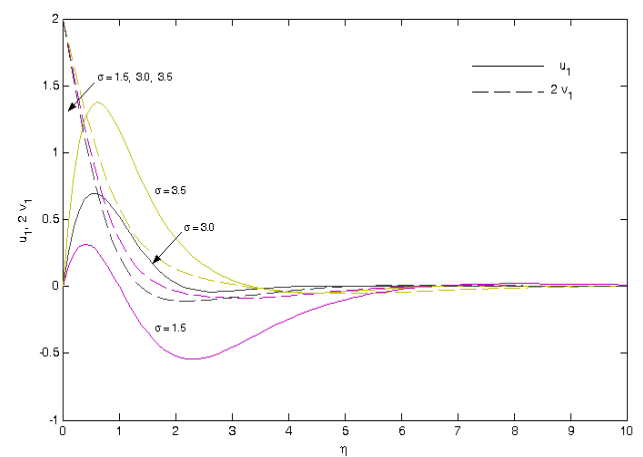

Figure 2. Velocities $u_{1}$ and $v_{1}$ for different $\sigma$ when

$$
k=0.05
$$

The transverse shearing stress on the disk $z=0$ is

$$
\begin{aligned}
\left(\tau_{z \theta}\right)_{z=0} & =\left[\rho\left(v-k_{0}^{*} \frac{\partial}{\partial t}\right) \frac{\partial u}{\partial z}\right]_{z=0}, \\
& =\left\{\begin{array}{l}
-\frac{1}{2} \rho r \varepsilon n^{*}(v \Omega)^{1 / 2} \\
\times\left[\left(\alpha_{1}+i \beta_{1}\right)+\left(\alpha_{2}+i \beta_{2}\right)\right] e^{i \sigma \tau} \text { for } \sigma>2 \\
-\frac{1}{2} \rho r \varepsilon n^{*}(v \Omega)^{1 / 2} \\
\times\left[\left(\alpha_{1}+i \beta_{1}\right)+\left(\alpha_{3}-i \beta_{3}\right)\right] e^{i \sigma \tau} \text { for } \sigma<2
\end{array}\right.
\end{aligned}
$$

(33)

The torque required to overcome this transverse shear stress on one side of the rotating disk of radius a (which is large enough to neglect the edge effects) is

$$
N=-\int_{0}^{a} r\left(\tau_{z \theta}\right)_{z=0}(2 \pi r) d r .
$$

Substituting the value of $\left(\tau_{z \theta}\right)_{z=0}$ from the equation (33) in the equation (34), we get

$$
N=R_{1} \cos (\sigma \tau+\theta)
$$

where

$$
\begin{gathered}
R_{1}=\frac{\pi \rho \varepsilon(2 v \Omega)^{\frac{1}{2}} a^{4}}{4}\left[\left(1+k^{2} \sigma^{2}\right)^{1 / 2}\left\{\sigma+\left(\sigma^{2}-4\right)^{\frac{1}{2}}\right\}\right]^{1 / 2} \\
\tan \theta=\frac{(a+b)-k \sigma(a-b)}{(a-b)+k \sigma(a+b)} \text { for } \sigma>2, \\
R_{1}=\frac{\pi \rho \varepsilon(2 v \Omega)^{\frac{1}{2}} a^{4}}{4}\left[\left(1+k^{2} \sigma^{2}\right)^{1 / 2}\left\{\left(\alpha_{1}+\alpha_{3}\right)^{2}+\left(\beta_{1}+\beta_{3}\right)^{2}\right\}^{1 / 2}\right] \\
\text { and } \tan \theta=\frac{\left(\beta_{1}-\beta_{3}\right)-k \sigma\left(\alpha_{1}+\alpha_{3}\right)}{\left(\alpha_{1}+\alpha_{3}\right)+k \sigma\left(\beta_{1}-\beta_{3}\right)} \text { for } \sigma<2
\end{gathered}
$$

\begin{tabular}{|c|c|c|c|c|c|}
\hline$\sigma \backslash k$ & 0.02 & 0.04 & 0.06 & 0.08 & 0.10 \\
\hline 0.5 & 2.00006 & 2.00021 & 2.00045 & 2.00080 & 2.00125 \\
\hline 1.0 & 2.00021 & 2.00080 & 2.00177 & 2.00319 & 2.00498 \\
\hline 1.5 & 2.00045 & 2.00180 & 2.00403 & 2.00716 & 2.01115 \\
\hline
\end{tabular}

It is interesting to note from (36) and (37) that the amplitude of the torque is affected by the elasticity of the fluid. It is seen from the equation (36) that $R_{1}$ increases with increase in either $k$ or $\sigma$ for $\sigma>2$. The values of $R_{1}$ for $\sigma<2$ have been given in Table 1 for different values of $k$ and $\sigma$. It is observed that $R_{1}$ increases with an increase in either $k$ or $\sigma$.

Table 1. Amplitude $R_{1}$ for different values of $k$ when

In Fig. 3 we have plotted $\tan \theta$ versus $k$ for various values of $\sigma$. We observe that the tangent of phase $\tan \theta$ decreases with an increase in either $\sigma$ or $k$. It may be noted that the torque has always a phase lead. 


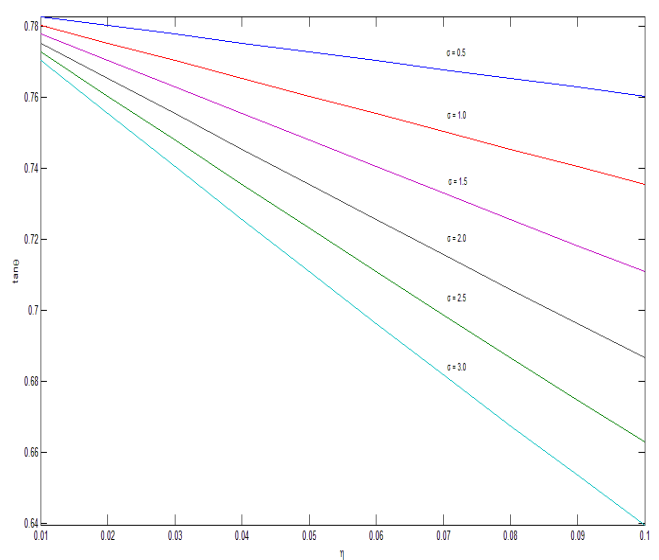

Figure 3. Tangent of phase $\tan \theta$ for different values of $\sigma$

From the equation (26), we get the axial velocity $w(\infty)$ as

$$
\frac{w(\infty)}{\varepsilon n^{*}(v / \Omega)^{\frac{1}{2}}}=R \sin (\sigma \tau-\phi),
$$

(38)

where the values of $R$ and $\phi$ are given by equations (27) and (28) for both the cases $\sigma>2$ and $\sigma<2$ respectively. It is seen from the equation (27) that the amplitude $R$ increases with an increase in $k$ when $\sigma>2$. Table 2 shows that $R$ increases with an increase in either $k$ or $\sigma$ for $\sigma<2$.

Table 2. Amplitude $R$ for different values of $k$ when $\sigma<2$

\begin{tabular}{|c|c|c|c|c|c|}
\hline$\sigma \backslash k$ & 0.02 & 0.04 & 0.06 & 0.08 & 0.10 \\
\hline 0.5 & 0.18430 & 0.18507 & 0.18635 & 0.18813 & 0.19038 \\
1.0 & 0.42301 & 0.42407 & 0.42583 & 0.42827 & 0.43136 \\
1.5 & 0.87980 & 0.88014 & 0.88071 & 0.88150 & 0.88251 \\
\hline
\end{tabular}

The values of phase $\phi$ have been plotted versus $k$ for several values of $\sigma$ in Figs.4 and 5. It is observed that for $\sigma<2$, the phase $\phi$ is always negative while for $\sigma>2$, the phase $\phi$ is always positive. Hence we conclude that the axial velocity $w(\infty)$ has a phase advance for $\sigma>2$ and a phase lag for $\sigma<2$.

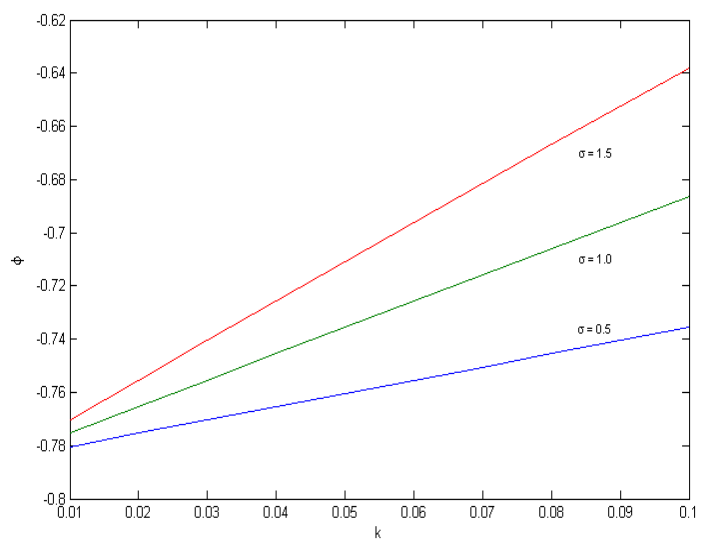

Figure 4. Phase $\phi$ for different values of $\sigma$

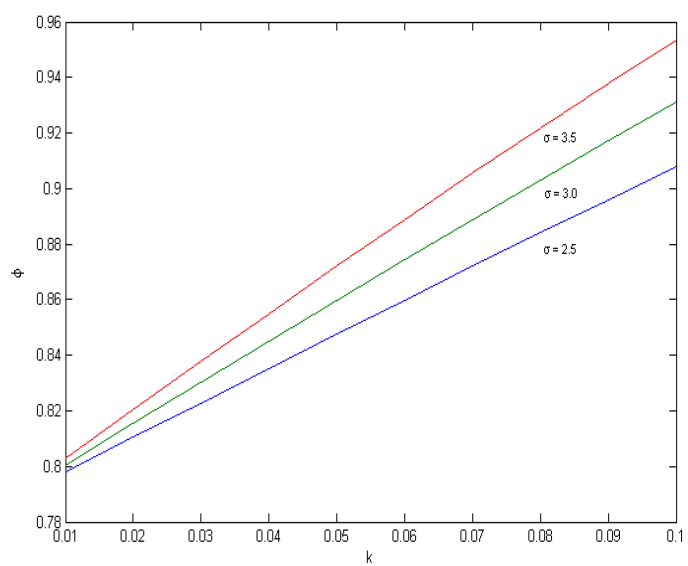

Figure 5. Phase $\phi$ for different values of $\sigma$

\section{REFERENCES}

[1] Rosenblat, S. (1959). Torsional oscillations of a plane in a viscous fluid, J. Fluid Mech., 6: 206.

[2] Henney, F. D. (1965). The flow induced by a disk oscillating about a state of steady rotation, Quart. J. Mech. Appl. Mech., 18: 333.

[3] Bhatnagar, P. L. and Rajeswari, C. K. (1963). The secondary flows induced in a non Newtonian flow between two parallel infinite oscillating planes, J. Indian Inst. Sci., 44(1962), pp.219.

[4] Srivastava, A. C. (1963). Torsional oscillations of an infinite plate is second order fluids, J. Fluid Mech., 17: 171.

[5] Walters, K. (1962). The solution of flow problems in the case of materials with memory, J. De Mech., 1: 474.

[6] Basu, U. and Debnath, L. (1976). Unsteady flows in an elastico-viscous fluid induced by torsional oscillation of a plate, Japan J. Appl. Phys., 15: 151.

[7] Rajagopal, K. R. (1992). Flow of visco-elastic fluids between rotating disks, Theor. Comput. Fluid Dyn., 3: 185-206.

[8] Thornly, C. (1968). On Stokes and Rayleigh layers in a rotating system, Quart. J. Mech, Appl. Math., 21: 451. 